\title{
Time Dependent Periodic Navier-Stokes Flows on a Two-Dimensional Torus
}

\author{
Zhi-Min Chen $^{\star 1}$, W.G. Price ${ }^{2}$ \\ 1 Department of Mathematics, Tianjin University, Tianjin 300072, P.R. China. E-mail: zhimin@tju.edu.cn \\ 2 Department of Ship Science, Southampton University, Southampton SO17 1BJ, UK
}

Received: 28 April 1995 / Accepted: 28 November 1995

\begin{abstract}
The dynamical behaviour of an incompressible viscous fluid flow on a twodimensional torus externally excited by a spatially periodic force is investigated. The flow field, described by Navier-Stokes equations, is found to possess a sequence of time-periodic solutions which bifurcate from a single steady state solution (i.e. Hopf bifurcations). This result is based on a combination of analysis and computations, and each provides corroborative evidence to the findings of the other.
\end{abstract}

\section{Introduction}

The Hopf bifurcation plays an important role in describing the behaviour of nonlinear phenomena occurring in the theory of dynamical systems (see for example, Marsden and McCracken (1976), Guckenheimer and Holmes (1983), Thompson and Stewart (1986)). In the flow of a viscous fluid, for example, it is commonly supposed to appear in transition from a steady state to a time dependent periodic flow. Landau (1944), Ruelle and Takens (1971), Newhouse et al. (1978) considered Hopf bifurcation to occur in the initial stage of transition from laminar to turbulent flows. However, from a strictly rigorous analytical viewpoint detailed studies of the existence of Hopf bifurcations in fluid motions are rather limited. The reason for this lies in the lack of efficient analysis methods to solve the problem, partly because the dynamical system modelling the fluid flow has an infinite number of degrees of freedom.

The Hopf bifurcation is central to the theme of this paper and through a combination of analysis with computation, we discuss its occurrence in an incompressible viscous fluid flow on a two-dimensional torus externally excited by a sinusoidal force. To do so, let $k \geq 1$ be a positive integer and $\mathscr{T}^{2}$ the two-dimensional torus $\mathscr{S}^{1} \times \mathscr{S}^{1}$ with $\mathscr{S}^{1}$ the unit circle $\mathscr{R} /(2 \pi \mathscr{Z})$. Thus, in an incompressible viscous fluid, we examine the behaviour of time dependent periodic Navier-Stokes flows excited by a spatially sinusoidal external body force $\left(4 k^{2} \sin 2 k y, 0\right)$.

The dynamical behaviour of this fluid flow system defined in terms of velocity $u=\left(u_{1}, u_{2}\right)$ and pressure $p$ is described by the Navier-Stokes equations

\footnotetext{
* Research partially supported by the National Natural Science Foundation of China.
} 


$$
\begin{array}{rlrl}
\partial_{t} u-\Delta u+\lambda u \cdot \nabla u+\nabla p & =\left(4 k^{2} \sin 2 k y, 0\right), & \\
\nabla \cdot u & =0, & \\
u(t, 0, y) & =u(t, 2 \pi, y), & & y \in[0,2 \pi), t \geq 0, \\
u(t, x, 0) & =u(t, x, 2 \pi), & & x \in[0,2 \pi), t \geq 0 .
\end{array}
$$

Here $\Delta$ and $\nabla$ denote the Laplacian and gradient operators respectively, $\partial_{s}=\partial / \partial s$ for $s=t, x$ and $y$, and $\lambda>0$ represents the Reynolds number defining the viscous fluid motion.

To ensure the uniqueness of the solution to Eq. (1), we require the additional condition

$$
\int_{\mathscr{T}^{2}} u(t, x, y) \mathrm{d} x \mathrm{~d} y=0, t \in(0, \infty) .
$$

The flow problem defined by these equations was first formulated by Kolmogorov (see, Arnold and Meshalkin (1960)), and is commonly referred to as the Kolmogorov problem (see, for example, Okamoto and Shoji (1991)).

Meshalkin and Sinai (1961) investigated the global stability of an incompressible fluid motion in a two-dimensional torus generated by an external sinusoidal force. From their study it follows that the global attractor of Eq. (1) with the force $\left(4 k^{2} \sin 2 k y, 0\right)$ replaced by $(\sin y, 0)$ consists of the only steady state solution $(\sin y, 0)$. Moreover, if we consider the force $\left(k^{\prime 2} \sin k^{\prime} y, 0\right)$ with $k^{\prime} \geq 2$ instead of $\left(4 k^{2} \sin 2 k y, 0\right)$ with $k \geq 1$, the existence of steady state solutions different to the simple one $\left(\sin k^{\prime} y, 0\right)$ can be obtained by following the approach of the study from Iudovich (1965). However, for the forcing assumed in this paper, it is found that there can also exist time dependent periodic solutions. Iudovich (1971) also derived a general existence result relating periodic solutions to the Navier-Stokes equations using the Lyapunov-Schmidt method. However, for the torus problem under discussion here, this result is difficult to apply because of the complexity of the eigenvalues associated with the linearized Navier-Stokes operator. Liu (1992a,b) examined instability of motion on the torus with reference to Hopf bifurcations, whereas Franceschini et al. (1988) and Okamoto and Shoji (1991), through numerical experiments, truncated the dynamical system modelling the fluid flow to one of finite dimensions. One can also refer to the study of Marchioro (1986) for an alternative approach confirming the result of Meshalkin and Sinai (1961).

In order to discuss the Hopf bifurcations and the subsequent time dependent periodic flows in the viscous fluid on the two-dimensional torus, this paper is organised as follows. In Sect. 2, we formulate the model describing the forced fluid motion, and prove that this problem has infinite invariant spaces. Therefore we reduce the fluid motion within a general space to one which is invariant with respect to Eq. (1) and this may contain periodic flows. In Sect. 3, we study the spectral problem of the linearized Navier-Stokes operator in $[\sqrt{3} k]$ different invariant spaces, and find, in each such a space, a non-real eigenvalue arising on both sides of the imaginary axis as the Reynolds number varies. Here $[a]$ denotes the integer part of $a$. This finding suggests that Eq. (1) admits $[\sqrt{3} k]$ Hopf bifurcation values. In Sect. 4 , to support this suggestion, we truncate the Navier-Stokes equations to $[\sqrt{3} k]$ coupled sets of five ordinary differential equations. Each set reveals Hopf bifurcations, and the bifurcated time dependent periodic orbits always appear to be limit cycle solutions for all Reynolds numbers. In Sect. 5, as an example, we provide computional results in the form of phase portraits for the limit cycle solution for the special case $k=1$. To provide additional evidence to confirm these theoretical and computational results, 
the Navier-Stokes dynamical system is truncated to a thirteen-dimensional dynamical system. (The latter is computationally manageable without excessive difficulty.) This extended investigation further corroborates the trends observed in the findings of Sects. 3-4.

In brief, this investigation shows that the time dependent Navier-Stokes flows excited by a prescribed spatially sinusoidal external force exhibit $[\sqrt{3} k]$ Hopf bifurcations and when the Reynolds number increases through each of these bifurcation values a periodic flow arises from the steady state solution. Furthermore, no second step bifurcation was observed in the flows as Reynolds number varies.

\section{Existence of Invariant Spaces}

It is easy to verify that $u=(\sin 2 k y, 0)$ is a steady state solution of Eq. (1). However, in this paper, we are interested in the existence of solutions bifurcated from $(\sin 2 k y, 0)$ and satisfying the time dependent periodic condition:

$$
u(t, x, y)=u(t+T, x, y),(t, x, y) \in(0, \infty) \times[0,2 \pi] \times[0,2 \pi]
$$

with period $T>0$.

To address this problem, it is more convenient to adopt the stream function $\psi$ rather than $u$ - with vorticity $\operatorname{curl} u=-\Delta \psi$ and velocity $u=\left(u_{1}, u_{2}\right)=\left(\partial_{y} \psi,-\partial_{x} \psi\right)$. This allows the elimination of the pressure from Eq. (1) which now takes the form

$$
\partial_{t} \Delta \psi-\Delta^{2} \psi+\lambda\left(\partial_{y} \psi \partial_{x} \Delta \psi-\partial_{x} \psi \partial_{y} \Delta \psi\right)=8 k^{3} \cos 2 k y
$$

associated with the modified condition

$$
\int_{\mathscr{T}^{2}} \psi(t, x, y) \mathrm{d} x \mathrm{~d} y=0
$$

ensuring uniqueness of solution. Note that both $(\lambda, \psi)$ and $(-\lambda,-\psi)$ satisfy Eq. (2) and therefore, if $\lambda_{0}$ is a bifurcation value so also is $-\lambda_{0}$.

To help formulate the discussion, the following spaces are adopted:

$$
\begin{aligned}
L^{2} & =\text { the } L^{2} \text { space }\left\{\psi:\left.\mathscr{T}^{2} \rightarrow \mathscr{R}\left|\|\psi\|^{2}=\int_{\mathscr{T}^{2}}\right| \psi\right|^{2} \mathrm{~d} x \mathrm{~d} y<\infty\right\} \\
W^{2,2} & =\text { the Sobolev space }\left\{\psi \in L^{2} \mid \Delta \psi \in L^{2}\right\} \\
H & =\text { the quotient space } L^{2} / \mathscr{R} \\
H^{2} & =\text { the quotient space } W^{2,2} / \mathscr{R} .
\end{aligned}
$$

Note that the multiplication $\phi \psi \in W^{2,2}$ whenever $\phi, \psi \in W^{2,2}$. This allows $H^{2}$ to be a Banach algebra by defining the product $\phi \cdot \psi=\phi \psi$ in $H^{2}$. Thus we can now introduce the following subspace of $H^{2}$ :

$$
\begin{aligned}
H_{l, k}^{2}= & \text { the Banach subalgebra of } H^{2} \text { generated by the three elements } \\
& \cos k y, \cos (l x-k y) \text { and } \cos (l x+k y),
\end{aligned}
$$

where $l$ is a positive integer.

Obviously, $H_{l^{\prime}, k}^{2} \subset H_{l, k}^{2}$ when $l^{\prime} / l \geq 1$ is an odd number, and furthermore there holds the following characterization: 


$$
\begin{aligned}
H_{l, k}^{2}= & \left\{\psi \in H^{2} \mid \psi=\sum_{m=1}^{\infty} \sum_{n=-\infty}^{\infty} \eta_{m, n} \cos (2 m l x-l x+2 n k y+k y)\right. \\
& \left.+\sum_{n=1}^{\infty} \xi_{n} \cos 2 n k y+\sum_{m=1}^{\infty} \sum_{n=-\infty}^{\infty} \zeta_{m, n} \cos (2 m l x+2 n k y)\right\} .
\end{aligned}
$$

Additionally, observing that the inverse $\Delta^{-1}: H \rightarrow H^{2}$ of $\Delta$ is continuous, we can rewrite Eqs. (2-3) in the form of a functional differential equation:

$$
\frac{\mathrm{d} \psi}{\mathrm{d} t}-\Delta \psi=f_{\lambda}(\psi)
$$

where

$$
f_{\lambda}(\psi)=-\lambda \Delta^{-1}\left(\partial_{y} \psi \partial_{x} \Delta \psi-\partial_{x} \psi \partial_{y} \Delta \psi\right)-2 k \cos 2 k y
$$

and $\psi(t)(\cdot, \cdot)=\psi(t, \cdot, \cdot) \in H^{2}$.

We thus seek Hopf bifurcations and periodic solutions of Eq. (4) in $H_{l, k}^{2}$, which are now proved to be invariant with respect to Eq. (4).

Let $e^{t \Delta}, t \geq 0$, be the contraction analytic semigroup in $H$ generated by the Laplacian. Since $e^{t \Delta} \cos (m x+n y)=e^{-\left(m^{2}+n^{2}\right) t} \cos (n x+m y)$, we see that

$$
e^{t \Delta} \psi, e^{t \Delta} f_{\lambda}(\psi) \in H_{l, k}^{2} \text { whenever } \psi \in H_{l, k}^{2} \text { and } t>0 .
$$

Thus we state the invariance result in a slightly general form.

Lemma 2.1. Let $H_{0}$ be a Banach subalgebra of $H^{2}$ such that

$$
e^{t \Delta} \psi, e^{t \Delta} f_{\lambda}(\psi) \in H_{0} \text { whenever } \psi \in H_{0} \text { and } t>0 .
$$

Then there exists a unique solution of Eq. (4) such that

$$
\psi \in C\left([0, \infty) ; H_{0}\right) \text { and } \psi(0)=\psi_{0}
$$

Proof. Note that for $\psi \in C\left([0, \infty) ; H^{2}\right)$, Eq. (4) is equivalent to the integral equation

$$
\psi(t)=e^{t \Delta} \psi_{0}+\int_{0}^{t} e^{(t-s) \Delta} f_{\lambda}(\psi) \mathrm{d} s
$$

To ease the subsequent discussion, let $F_{\psi_{0}}(\psi)$ denote the right-hand side of this integral equation with $F_{\psi_{0}}$ representing an operator.

To study the fixed point problem of this operator, let us begin by introducing a local existence argument. Given $\delta>0, K>0$, and defining

$$
W\left(\delta, K, \psi_{0}\right)=\left\{\psi \in C\left([0, \delta] ; H_{0}\right) \mid\left\|\psi-\psi_{0}\right\|_{W}=\max _{0 \leq t \leq \delta}\left\|\Delta \psi-\Delta \psi_{0}\right\| \leq K\right\}
$$

we have for $\psi \in W\left(\delta, K, \psi_{0}\right), 0 \leq t \leq \delta$ and a generic constant $c>0$,

$$
\left\|\Delta F_{\psi_{0}} \psi(t)-\Delta e^{t \Delta} \psi_{0}\right\|
$$




$$
\begin{aligned}
& =\left\|\int_{0}^{t}\left\{\partial_{x} e^{(t-s) \Delta}\left(\partial_{y} \psi \Delta \psi\right)-\partial_{y} e^{(t-s) \Delta}\left(\partial_{x} \psi \Delta \psi\right)-8 k^{3} e^{(t-s) \Delta} \cos 2 k y\right\} \mathrm{d} s\right\| \\
& \leq c \int_{0}^{t}(t-s)^{-3 / 4}\left(\left\|\partial_{y} \psi \Delta \psi\right\|_{L^{4 / 3}}+\left\|\partial_{x} \psi \Delta \psi\right\|_{L^{4 / 3}}\right) \mathrm{d} s+16 k^{3} \pi t \\
& \leq c \int_{0}^{t}(t-s)^{-3 / 4}\left(\left\|\partial_{y} \psi\right\|_{L^{4}}+\left\|\partial_{x} \psi\right\|_{L^{4}}\right)\|\Delta \psi\| \mathrm{d} s+16 k^{3} \pi t \\
& \leq c K^{2} t^{1 / 4}+16 k^{3} \pi t \leq c K^{2} \delta^{1 / 4}+16 k^{3} \pi \delta
\end{aligned}
$$

where use is made of the Hölder inequality, the $L^{p}-L^{q}$ estimates and the Sobolev imbedding inequality (see, for example, Triebel (1978)). Similarly, we have for $\psi, \phi \in$ $W\left(\delta, K, \psi_{0}\right)$ and $0 \leq t \leq \delta$,

$$
\left\|\Delta F_{\psi_{0}} \psi(t)-\Delta F_{\psi_{0}} \phi(t)\right\| \leq c K t^{1 / 4} \sup _{0<t<\delta}\|\Delta \psi(t)-\Delta \phi(t)\| \leq c K \delta^{1 / 4}\|\psi-\phi\|_{W}
$$

Thus, by Eq. (5) and the strong continuity of $e^{t \Delta}$, we find that $F_{\psi_{0}}$ is a contraction operator mapping $W\left(\delta, K, \psi_{0}\right)$ into itself, provided that $\delta$ is sufficiently small. Therefore, the Banach fixed point theorem implies the uniqueness and existence of the solution to Eq. (4) in $W\left(\delta, K, \psi_{0}\right)$.

Additionally, if $\psi \in C\left([0, T) ; H_{0}\right)$ satisfies Eq. (4) for some $T>0$, we see that the solution $\psi$ is smooth. Multiplying Eq. (4) by $\Delta^{2} \psi$ and integrating over $\mathscr{T}^{2}$, we obtain

$$
\frac{\mathrm{d}\|\Delta \psi\|^{2}}{\mathrm{~d} t}+2\|\nabla \Delta \psi\|^{2} \leq 2\left\|4 k^{2} \cos 2 k y\right\|\|\nabla \Delta \psi\|
$$

and so

$$
\frac{\mathrm{d}\|\Delta \psi\|^{2}}{\mathrm{~d} t}+\|\Delta \psi\|^{2} \leq\left\|4 k^{2} \cos 2 k y\right\|^{2}=32 k^{4} \pi^{2}
$$

and hence for $0 \leq t<T$,

$$
\|\Delta \psi(t)\|^{2} \leq e^{-t}\|\Delta \psi(0)\|^{2}+32 k^{4} \pi^{2} \leq\|\Delta \psi(0)\|^{2}+32 k^{4} \pi^{2} .
$$

Through the local solution extension procedure, we arrive at the desired conclusion, and the proof is complete.

With this lemma, we may reduce Eq. (4) defined in space $H^{2}$ to the subspaces $H_{l, k}^{2}$ and thus greatly simplify the problem. More specifically, we seek time dependent periodic solutions of the equation

$$
\frac{\mathrm{d} \psi}{\mathrm{d} t}=\Delta \psi+f_{\lambda}(\psi), \quad \psi(t) \in H_{l, k}^{2}
$$

for $1 \leq l<\sqrt{3} k$. (The imposition of this latter restriction on $l$ is discussed in the following section.) 


\section{Spectral Problem}

Let

$$
A_{\lambda}=\Delta-\lambda \Delta^{-1}\left(\sin 2 k y\left(\Delta+4 k^{2}\right)\right) \partial_{x}
$$

which is the Fréchet derivative of $\Delta \psi+f_{\lambda}(\psi)$ with respect to the steady state solution $-(1 / 2 k) \cos 2 k y$. We note that $A_{\lambda}$ contains the operator $\Delta+4 k^{2}$, which, in fact, allows the existence of non-real eigenvalues across the imaginary axis as $\lambda$ increases. A Hopf bifurcation is essentially based on such an observation (see, for example, Guckenheimer and Holmes (1983)). This section is devoted to the determination of the eigenvalues of the operator $A_{\lambda}$ in $H_{l, k}^{2}$, which, for convenience, is supposed to be a complex space in this section.

Theorem 3.1. For every $\lambda>0$, the spectral problem

$$
A_{\lambda} \psi=\rho(\lambda) \psi \text {. }
$$

in $H_{l, k}^{2}$ has no eigenvalues with nonnegative real parts provided that the integer $l>$ $\sqrt{3} k$, and it has an eigenvalue $\rho_{l}(\lambda)$ with $\operatorname{Im} \rho_{l}(\lambda) \neq 0$ such that

$$
\lim _{\lambda \rightarrow 0} \rho_{l}(\lambda)=-l^{2}-k^{2}, \quad \operatorname{Re} \rho_{l}\left(\lambda_{l}\right)=0, \operatorname{Re} \rho_{l}(\lambda)>0\left(\lambda_{l}<\lambda<\lambda_{l}+\delta\right)
$$

for some positive constants $\lambda_{l}$ and $\delta$, provided that $1 \leq l<\sqrt{3} k$.

Proof. Firstly, we derive an alternative form of Eq. (7) and prove the assertion in the case $l>\sqrt{3} k$. Observing that

$$
\hat{H}_{l, k}^{2}=\left\{\psi \in H_{l, k}^{2} \mid \psi=\sum_{m=-\infty}^{\infty} \xi_{m} \cos (l x+2 m k y+k y)\right\}
$$

is an invariant space with respect to the operator $A_{\lambda}$ for every $l \geq 1$, we may consider Eq. (7) in $\hat{H}_{l, k}^{2}$. That is, we reduce the spectral problem of the operator $A_{\lambda}$ in $H_{l, k}^{2}$ to that in $\hat{H}_{l, k}^{2}$. Letting $\mathscr{C}$ be the complex plane, and $(\rho, \psi) \in \mathscr{C} \times\left(\hat{H}_{l, k}^{2} \backslash\{0\}\right)$ be a solution to Eq. (7) with

$$
\psi=\sum_{m=-\infty}^{\infty} \xi_{l, m} \cos (l x+2 m k y+k y)
$$

we have for $m \in \mathscr{Z}$,

$$
\begin{aligned}
0= & \frac{1}{2 \pi^{2}} \int_{\mathscr{T}^{2}}\left(A_{\lambda} \psi-\rho \psi\right) \cos (l x+2 m k y+k y) \mathrm{d} x \mathrm{~d} y \\
= & -\left(l^{2}+(2 m k+k)^{2}+\rho\right) \xi_{l, m}+\frac{1}{2 \pi^{2}} \lambda \sum_{n=-\infty}^{\infty} l\left(l^{2}+(2 n k+k)^{2}-4 k^{2}\right) \xi_{l, n} \\
& \cdot \int_{\mathscr{T}^{2}} \cos (l x+2 m k y+k y) \Delta^{-1}\{\sin 2 k y \sin (l x+2 n k y+k y)\} \mathrm{d} x \mathrm{~d} y \\
= & -\left(l^{2}+(2 m k+k)^{2}+\rho\right) \xi_{l, m}+\frac{\lambda l\left(l^{2}+(2(m+1) k+k)^{2}-4 k^{2}\right)}{2\left(l^{2}+(2 m k+k)^{2}\right)} \xi_{l, m+1} \\
& -\frac{\lambda l\left(l^{2}+(2(m-1) k+k)^{2}-4 k^{2}\right)}{2\left(l^{2}+(2 m k+k)^{2}\right)} \xi_{l, m-1} \\
= & -\left(\beta_{l, m}+\rho\right) \xi_{l, m}+\lambda \frac{\alpha_{l, m+1} \xi_{l, m+1}}{2 \beta_{l, m}}-\lambda \frac{\alpha_{l, m-1} \xi_{l, m-1}}{2 \beta_{l, m}}
\end{aligned}
$$


where

$$
\beta_{l, m}=l^{2}+(2 m k+k)^{2} \text { and } \alpha_{l, m}=l\left(l^{2}+(2 m k+k)^{2}-4 k^{2}\right) .
$$

Following the reasoning of Meshalkin and Sinai (1961), it is easy to verify that $\xi_{l, m} \neq 0$ for all $m \in \mathscr{Z}$. Thus by defining

$$
\begin{aligned}
\gamma_{l, m} & =\frac{\alpha_{l, m} \xi_{l, m}}{\alpha_{l, m-1} \xi_{l, m-1}} \text { for } m \geq 0 \\
\gamma_{l, m} & =\frac{\alpha_{l, m} \xi_{l, m}}{\alpha_{l, m+1} \xi_{l, m+1}} \text { for } m<0
\end{aligned}
$$

and using the fact $\psi \in H_{l, k}^{2}$, we obtain the boundedness of $\gamma_{l, m}$ with respect to $m \in \mathscr{Z}$. It follows from Eq. (9) that

$$
\begin{aligned}
\gamma_{l, m} & =\frac{-1}{\frac{2\left(\beta_{l, m}+\rho\right) \beta_{l, m}}{\lambda \alpha_{l, m}}-\gamma_{l, m+1}} \text { for } m \geq 0, \\
\gamma_{l, m} & =\frac{1}{\frac{2\left(\beta_{l, m}+\rho\right) \beta_{l, m}}{\lambda \alpha_{l, m}}+\gamma_{l, m-1}} \text { for } m<0 .
\end{aligned}
$$

We thus have $\gamma_{l, m} \rightarrow 0$ as $m \rightarrow \pm \infty$, and so

$$
\begin{gathered}
\gamma_{l, m}=\frac{-1}{\frac{2\left(\beta_{l, m}+\rho\right) \beta_{l, m}}{\lambda \alpha_{l, m}}+\frac{1}{\frac{2\left(\beta_{l, m+1}+\rho\right) \beta_{l, m+1}}{\lambda \alpha_{l, m+1}}+\frac{1}{\ddots}}} \text { for } m \geq 0, \\
\gamma_{l, m}=\frac{1}{\frac{2\left(\beta_{l, m}+\rho\right) \beta_{l, m}}{\lambda \alpha_{l, m}}+\frac{1}{\frac{2\left(\beta_{l, m-1}+\rho\right) \beta_{l, m-1}}{\lambda \alpha_{l, m-1}}+\frac{1}{\ddots}}} \text { for } m<0,
\end{gathered}
$$

and hence $\gamma_{l, 0}=-\gamma_{l,-1}$ since Eq. (10) implies $\beta_{l,-m}=\beta_{l, m-1}$ and $\alpha_{l,-m}=\alpha_{l, m-1}$ for $m \geq 1$. On the other hand, by Eq. (11), $\gamma_{l, 0}=1 / \gamma_{l,-1}$, and so $\gamma_{l, 0}^{2}=-1$. We note that if $\rho$ satisfies the equation $\gamma_{l, 0}=i, \bar{\rho}$ satisfies the equation $\gamma_{l, 0}=-i$. Therefore, Eq. (7) becomes the equation $\gamma_{l, 0}=i$, or by Eq. (12) with $m=0$,

$$
\frac{2\left(\beta_{l, 0}+\rho\right) \beta_{l, 0}}{\lambda \alpha_{l, 0}}+\frac{1}{\frac{2\left(\beta_{l, 1}+\rho\right) \beta_{l, 1}}{\lambda \alpha_{l, 1}}+\frac{1}{\frac{2\left(\beta_{l, 2}+\rho\right) \beta_{l, 2}}{\lambda \alpha_{l, 2}}+\frac{1}{\ddots}}}-i=0 .
$$

From Eq. (10), we see $\beta_{l, m}>0$ and $\alpha_{l, m+1}>0$ for all $l \geq 1$, and $m \geq 0$. Thus, if this equation has a solution with $\operatorname{Re} \rho \geq 0$, then

$$
\alpha_{l, 0}=l\left(l^{2}-3 k^{2}\right)<0 .
$$


This is impossible if $l>\sqrt{3} k$. Moreover, for such an integer $l$, it is readily seen that Eq. (7) in $\mathscr{C} \times H_{l, k}^{2}$ has no other non-trivial solution $(\rho, \psi)$ with $\operatorname{Re} \rho \geq 0$. This shows the assertion in the case $l>\sqrt{3} k$, and hence we derive the condition, $l<\sqrt{3} k$, imposed in Eq. (6).

Secondly, we show the existence of the eigenvalue $\rho$ with $\operatorname{Re} \rho>-\beta_{l, 0}$ and $\operatorname{Im} \rho \neq 0$ when $1 \leq l<\sqrt{3} k . \operatorname{Im} \rho \neq 0$ is obviously valid if $\rho$ satisfies Eq. (14). Multiplying Eq. (14) by $\lambda \alpha_{l, 0} / 2 \beta_{l, 0}$ yields

$$
-\beta_{l, 0}+i \frac{\alpha_{l, 0} \lambda}{2 \beta_{l, 0}}-\frac{\alpha_{l, 0} / 2 \beta_{l, 0}}{\frac{2\left(\beta_{l, 1}+\rho\right) \beta_{l, 1}}{\lambda^{2} \alpha_{l, 1}}+\frac{1}{\frac{2\left(\beta_{l, 2}+\rho\right) \beta_{l, 2}}{\alpha_{l, 2}}+\frac{1}{\ddots}}}=\rho .
$$

Let

$$
\mu=\operatorname{Re} \rho, \nu=\operatorname{Im} \rho, \quad \text { and } \Phi_{\lambda, l}(\mu, \nu)=\left(\operatorname{Re} \Psi_{\lambda, l}(\mu, \nu), \operatorname{Im} \Psi_{\lambda, l}(\mu, \nu)\right),
$$

where $\Psi_{\lambda, l}(\mu, \nu)$ denotes the left-hand side of Eq. (15). This equation is solved when a suitable value is found for $(\mu, \nu)=\left(\mu_{l}(\lambda), \nu_{l}(\lambda)\right)$, a fixed point of $\Phi_{\lambda, l}$.

Note that $1 \leq l<\sqrt{3} k$ implies $\alpha_{l, 0}<0$, which yields

$$
\operatorname{Re} \Psi_{\lambda, l}(\mu, \nu)>-\beta_{l, 0},
$$

and

$$
\left|\Psi_{\lambda, l}(\mu, \nu)+\beta_{l, 0}\right| \leq \frac{\left|\alpha_{l, 0}\right| \lambda}{2 \beta_{l, 0}}+\frac{\left|\alpha_{l, 0}\right| \alpha_{l, 1} \lambda^{2}}{4 \beta_{l, 0} \beta_{l, 1}\left(\beta_{l, 1}+\mu\right)}
$$

for all $\mu \geq-\beta_{l, 0}$ and $\nu \in \mathscr{R}$. Setting the constant

$$
K(\lambda)=\frac{\left|\alpha_{l, 0}\right| \lambda}{2 \beta_{l, 0}}+\frac{\left|\alpha_{l, 0}\right| \alpha_{l, 1} \lambda^{2}}{4 \beta_{l, 0} \beta_{l, 1}\left(\beta_{l, 1}-\beta_{l, 0}\right)},
$$

we have

$$
\Phi_{\lambda, l}:\left[-\beta_{l, 0}, K\right] \times[-K, K] \rightarrow\left[-\beta_{l, 0}, K\right] \times[-K, K] .
$$

Since $\Phi_{\lambda, l}$ is a continuous function and the set $\left[-\beta_{l, 0}, K\right] \times[-K, K]$ is homeomorphic to the unit disk of $\mathscr{R}^{2}$, by the Brouwer fixed-point theorem (see, for example, Massey (1991)), $\Phi_{\lambda, l}$ admits a fixed point $\left(\mu_{l}(\lambda), \nu_{l}(\lambda)\right) \in\left[-\beta_{l, 0}, K\right] \times[-K, K]$ or

$$
\begin{aligned}
& \Psi_{\lambda, l}\left(\mu_{l}(\lambda), \nu_{l}(\lambda)\right)=\mu_{l}(\lambda)+i \nu_{l}(\lambda) \\
& =i \frac{\alpha_{l, 0} \lambda}{2 \beta_{l, 0}}-\beta_{l, 0}-\frac{\alpha_{l, 0} / 2 \beta_{l, 0}}{\frac{2\left(\beta_{l, 1}+\mu_{l}(\lambda)+i \nu_{l}(\lambda)\right) \beta_{l, 1}}{\lambda^{2} \alpha_{l, 1}}+\frac{1}{\frac{2\left(\beta_{l, 2}+\mu_{l}(\lambda)+i \nu_{l}(\lambda)\right) \beta_{l, 2}}{\alpha_{l, 2}}+\frac{1}{\ddots}}} .
\end{aligned}
$$

Finally, it remains to prove the validity of Eq. (8) for $\rho_{l}(\lambda)=\mu_{l}(\lambda)+i \nu_{l}(\lambda)$. Observing that $\lim _{\lambda \rightarrow 0} K(\lambda)=0$, we have

$$
\Psi_{\lambda, l}\left(\mu_{l}(\lambda), \nu_{l}(\lambda)\right) \rightarrow-\beta_{l, 0} \text { as } \lambda \rightarrow 0,
$$

and by Eq. (16), $\mu_{l}(\lambda) \rightarrow-\beta_{l, 0}<0$ as $\lambda \rightarrow 0$. Additionally, let us suppose that, on the contrary, $\mu_{l}(\lambda) \leq 0$ for all $\lambda>0$. It is not difficult to verify that $\nu_{l}(\lambda)=O(\lambda)$ as $\lambda$ tends to infinity by using Eq. (16) and the fact $\lim _{m \rightarrow \infty} \gamma_{l, m}=0$. Thus for $\lambda$ sufficiently large, it is valid that $\operatorname{Re} \Psi_{\lambda, l}\left(\mu_{l}(\lambda), \nu_{l}(\lambda)\right)>0$, and so $\mu_{l}(\lambda)>0$ by Eq. (16). This leads to a contradiction, and completes the proof. 


\section{Hopf Bifurcations}

From Theorem 3.1 we see that a pair of conjugate eigenvalues of $A_{\lambda}$ arise in the right half complex plane as the Reynolds number increases across $\lambda_{l}$ for every $1 \leq l<$ $\sqrt{3} k$, and the corresponding eigenfunctions are in the space $\hat{H}_{l, k}^{2}$. This provides limited evidence for the existence of Hopf bifurcations in the infinite-dimensional dynamical system represented by Eq. (6), and thus the existence of periodic Navier-Stokes flows in the spaces $H_{l, k}^{2}$. Although a rigorous mathematical proof of these statements is not available, fortunately by numerical experiment they can be substantiated. For example, by truncating Eq. (6) with $1 \leq l<\sqrt{3} k$ into a coupled set of ordinary differential equations, a Hopf bifurcation with respect to bifurcation value $\lambda_{l}$ can be demonstrated.

Let us begin with an observation to strengthen credence in the proposed truncation scheme. Since the Navier-Stokes flow is excited by a vector field in $\operatorname{span}\{\cos 2 k y\}$, and the spectral problem studied in Sec. 3 is only considered in the space $\hat{H}_{l, k}^{2}$, therefore $\hat{H}_{l, k}^{2} \times \operatorname{span}\{\cos 2 k y\}$ is the key subspace to investigate the Hopf bifurcation problem. Moreover, suppose that

$$
\psi=\sum_{m=-\infty}^{\infty} \xi_{l, m} \cos (l x+2 m k+k y)
$$

is an eigenfunction of $A_{\lambda}$ associated with the eigenvalue $\rho_{l}(\lambda)$. It therefore follows from Eq. (9) that

$$
\left(l^{2}+(2 m k+k)^{2}+\rho\right)\left(l^{2}+(2 m k+k)^{2}\right) \xi_{l, m} \rightarrow 0 \text { as } m \rightarrow \infty
$$

and hence for every $n>0$,

$$
(2 m k+k)^{n} \xi_{l, m} \rightarrow 0 \text { as } m \rightarrow \infty .
$$

This suggests that the modes $\cos (l x+2 m k y+k y)$ for $m$ large have little influence on the bifurcation problem. From Eqs. (12-13) we see that the mode $\cos (l x+2 m k y+k y)$ exhibits limited symmetry with the mode $\cos (l x-2 m k y-k y)$ for every $m \geq 0$ in the spectral problem examined in Sec. 3. Furthermore, it is easy to verify that the projection of Eq. (6) onto the space

$$
\operatorname{span}\{\cos 2 k y, \cos (l x-k y), \cos (l x+k y)\}
$$

produces a coupled set of three equations, which is globally stable and hence, no bifurcation is possible. It also follows that the simplest construction onto which the truncation can produce a coupled set of equations exhibiting Hopf bifurcations is the five-dimensional space

$$
\operatorname{span}\{\cos 2 k y, \cos (l x-3 k y), \cos (l x-k y), \cos (l x+k y), \cos (l x+3 k y)\} .
$$

The projection of Eq. (6) onto this space allows the Navier-Stokes flow defined by Eq. (6) to be truncated in the following manner:

$$
\psi=X_{1}(t) \cos 2 k y+\sum_{n=2}^{5} X_{n}(t) \cos (l x+2 n k y-7 k y)
$$


and with this representation, we find that the nonlinear term can be expressed in the form

$$
\begin{aligned}
\partial_{y} \psi \partial_{x} \Delta \psi- & \partial_{x} \psi \partial_{y} \Delta \psi \\
= & \sum_{n=2}^{4} k l\left(4 k^{2}-l^{2}-(2 n k-5 k)^{2}\right) X_{1} X_{n+1} \cos (l x+2 n k y-7 k y) \\
& -\sum_{n=3}^{5} k l\left(4 k^{2}-l^{2}-(2 n k-9 k)^{2}\right) X_{1} X_{n-1} \cos (l x+2 n k y-7 k y) \\
& +\sum_{n=3}^{5} 8 k^{3} l(n-4) X_{n} X_{n-1} \cos 2 k y+E
\end{aligned}
$$

where $E$ is a term orthogonal to the five-dimensional space. Thus the set of truncated equations

$$
\int_{\mathscr{T}^{2}}\left(\frac{\mathrm{d} \psi}{\mathrm{d} t}-\Delta \psi-f_{\lambda}(\psi)\right) \phi \mathrm{d} x \mathrm{~d} y=0, \phi=\cos 2 k y, \cos (l x+2 n k y-7 k y), 2 \leq n \leq 5
$$

gives

$$
\begin{aligned}
\frac{\mathrm{d} X_{1}}{\mathrm{~d} t} & =-4 k^{2} X_{1}+2 \lambda l k X_{2} X_{3}-2 \lambda l k X_{4} X_{5}-2 k \\
\frac{\mathrm{d} X_{2}}{\mathrm{~d} t} & =-\left(l^{2}+9 k^{2}\right) X_{2}-\lambda l k \frac{3 k^{2}-l^{2}}{9 k^{2}+l^{2}} X_{1} X_{3} \\
\frac{\mathrm{d} X_{3}}{\mathrm{~d} t} & =-\left(l^{2}+k^{2}\right) X_{3}-\lambda l k \frac{3 k^{2}-l^{2}}{k^{2}+l^{2}} X_{1} X_{4}-\lambda l k \frac{5 k^{2}+l^{2}}{k^{2}+l^{2}} X_{1} X_{2} \\
\frac{\mathrm{d} X_{4}}{\mathrm{~d} t} & =-\left(l^{2}+k^{2}\right) X_{4}+\lambda l k \frac{3 k^{2}-l^{2}}{k^{2}+l^{2}} X_{1} X_{3}+\lambda l k \frac{5 k^{2}+l^{2}}{k^{2}+l^{2}} X_{1} X_{5} \\
\frac{\mathrm{d} X_{5}}{\mathrm{~d} t} & =-\left(l^{2}+9 k^{2}\right) X_{5}+\lambda l k \frac{3 k^{2}-l^{2}}{9 k^{2}+l^{2}} X_{1} X_{4} .
\end{aligned}
$$

Let us examine this coupled set of equations to find a supercritical Hopf bifurcation value $\lambda_{l}^{*}$ approximating the true bifurcation value $\lambda_{l}$ of Eq. (6). When $\lambda$ increases across $\lambda_{l}^{*}$, a limit cycle solution arises from the equilibrium point $(-1 / 2 k, 0,0,0,0)$, which corresponds to the steady state solution $-(1 / 2 k) \cos 2 k y$ of Eq. (6).

For simplicity, let

$$
a=l^{2}+k^{2}, b=9 k^{2}+l^{2}, c=3 k^{2}-l^{2} \text { and } d=5 k^{2}+l^{2},
$$

and Eq. (17) becomes

$$
\begin{aligned}
\frac{\mathrm{d} X_{1}}{\mathrm{~d} t} & =-2 k\left(2 k X_{1}+1\right)+2 \lambda l k\left(X_{2} X_{3}-X_{4} X_{5}\right) \\
\frac{\mathrm{d} X_{2}}{\mathrm{~d} t} & =-b X_{2}-\frac{\lambda l k c}{b} X_{1} X_{3} \\
\frac{\mathrm{d} X_{3}}{\mathrm{~d} t} & =-a X_{3}-\frac{\lambda l k c}{a} X_{1} X_{4}-\frac{\lambda l k d}{a} X_{1} X_{2}, \\
\frac{\mathrm{d} X_{4}}{\mathrm{~d} t} & =-a X_{4}+\frac{\lambda l k c}{a} X_{1} X_{3}+\frac{\lambda l k d}{a} X_{1} X_{5}, \\
\frac{\mathrm{d} X_{5}}{\mathrm{~d} t} & =-b X_{5}+\frac{\lambda l k c}{b} X_{1} X_{4} .
\end{aligned}
$$


Denoting $B_{\lambda, l}(\mathbf{X})$ to represent the Fréchet derivative of the right hand side term of Eqs. (19-23) with respect to $\mathbf{X}=\left(X_{1}, X_{2}, X_{3}, X_{4}, X_{5}\right)$, we see that $B_{\lambda, l}(\mathbf{X})$ represents the matrix

$$
\left(\begin{array}{ccccc}
-4 k^{2} & 2 \lambda l k X_{3} & 2 \lambda l k X_{2} & -2 \lambda l k X_{5} & -2 \lambda l k X_{4} \\
-\frac{\lambda l k c X_{3}}{b} & -b & -\frac{\lambda l k c X_{1}}{b} & 0 & 0 \\
-\frac{\lambda l k\left(c X_{4}+d X_{2}\right)}{a} & -\frac{\lambda l k d X_{1}}{a} & -a & -\frac{\lambda l k c X_{1}}{a} & 0 \\
\frac{\lambda l k\left(c X_{3}+d X_{5}\right)}{a} & 0 & \frac{\lambda l k c X_{1}}{a} & -a & \frac{\lambda l k d X_{1}}{a} \\
\frac{\lambda l k c X_{4}}{b} & 0 & 0 & \frac{\lambda l k c X_{1}}{b} & -b
\end{array}\right) .
$$

Specifically, we have

$$
B_{\lambda, l}((-1 / 2 k, 0,0,0,0))=\left(\begin{array}{ccccc}
-4 k^{2} & 0 & 0 & 0 & 0 \\
0 & -b & \frac{\lambda l c}{2 b} & 0 & 0 \\
0 & \frac{\lambda l d}{2 a} & -a & \frac{\lambda l c}{2 a} & 0 \\
0 & 0 & -\frac{\lambda l c}{2 a} & -a & -\frac{\lambda l d}{2 a} \\
0 & 0 & 0 & -\frac{\lambda l c}{2 b} & -b
\end{array}\right)
$$

with characteristic polynomial

$$
\begin{aligned}
0 & =\operatorname{det}\left(B_{\lambda}((-1 / 2 k, 0,0,0,0))-\rho I\right) \\
& =-\left(4 k^{2}+\rho\right)\left(\left((a+\rho)(b+\rho)-\frac{\lambda^{2} l^{2} c d}{4 a b}\right)^{2}+\left(\frac{\lambda l c(b+\rho)}{2 a}\right)^{2}\right) .
\end{aligned}
$$

Let us examine the complex eigenvalues of this matrix with $\rho=\mu+i \nu$, and assume $\mu \geq 0$ is a root of this polynomial. We see that the eigenvalue satisfies the equation

$$
(a+\mu+i \nu)(b+\mu+i \nu)-\frac{\lambda^{2} l^{2} c d}{4 a b}-i \frac{\lambda l c(b+\mu+i \nu)}{2 a}=0
$$

giving

$$
\begin{aligned}
a b+2 d \mu+\mu^{2}-\nu^{2}-\frac{\lambda^{2} l^{2} c d}{4 a b}+\frac{\lambda l c \nu}{2 a} & =0, \\
2 d \nu+2 \mu \nu-\frac{\lambda l c(b+\mu)}{2 a} & =0
\end{aligned}
$$

and so

$$
\nu=\frac{\lambda l c(b+\mu)}{4 a(d+\mu)} .
$$

After manipulation, these equations give

$$
4(d+\mu)^{2}\left((a+\mu)(b+\mu)-\frac{\lambda^{2} l^{2} c d}{4 a b}\right)+\frac{\lambda^{2} l^{2} c^{2}(b+\mu)(a+\mu)}{4 a^{2}}=0
$$


providing an equation in $\mu$ only. Since a Hopf bifurcation, with bifurcation value $\lambda=\lambda_{l}^{*}$, can only occur when the real part $\mu$ is near to zero, Eq. (24) implies that

$$
\lambda_{l}^{*}=\frac{4 a b d}{l \sqrt{4 c d^{3}-c^{2} b^{2}}}=\frac{4\left(k^{2}+l^{2}\right)\left(9 k^{2}+l^{2}\right)\left(5 k^{2}+l^{2}\right)}{l \sqrt{4\left(3 k^{2}-l^{2}\right)\left(5 k^{2}+l^{2}\right)^{3}-\left(3 k^{2}-l^{2}\right)^{2}\left(9 k^{2}+l^{2}\right)^{2}}}
$$

when $\mu=0$. Thus, if $\eta_{l}(\mu, \lambda)$ represents the left-hand side of Eq. (24), then at $\mu=0$, we have

$$
\begin{aligned}
\eta_{l}\left(0, \lambda_{l}^{*}\right) & =0, \\
\frac{1}{2 d} \frac{\partial \eta_{l}\left(0, \lambda_{l}^{*}\right)}{\partial \mu} & =4 a b+a^{2} b^{2}-\frac{\lambda_{l}^{* 2} l^{2} c d}{a b}+\frac{\lambda_{l}^{* 2} l^{2} c^{2}}{4 a^{2}}>0
\end{aligned}
$$

and

$$
\frac{\partial \eta_{l}\left(0, \lambda_{l}^{*}\right)}{\partial \lambda}=\frac{\lambda_{l}^{*} l^{2} c\left(4 d^{3}-c b\right)}{8 a b d^{2}}<0
$$

by Eqs. $(25,18)$. Furthermore, by the implicit function theorem, it follows that $\mu=$ $\mu_{l}(\lambda)$ is the only function defined on a small interval $\left(\lambda_{l}^{*}-\delta, \lambda_{l}^{*}+\delta\right)$ such that

$$
\eta_{l}\left(\mu_{l}(\lambda), \lambda\right)=0, \mu_{l}\left(\lambda_{l}^{*}\right)=0, \frac{\mathrm{d} \mu_{l}\left(\lambda_{l}^{*}\right)}{\mathrm{d} \lambda}=-\frac{(\partial / \partial \lambda) \eta_{l}\left(0, \lambda_{l}^{*}\right)}{(\partial / \partial \mu) \eta_{l}\left(0, \lambda_{l}^{*}\right)}>0 .
$$

These results can now be summarised as follows:

Lemma 4.1. For every $l=1, \ldots,[\sqrt{3} k]$, there exists a unique positive number $\lambda_{l}^{*}$ associated with an eigenvalue $\rho_{l}^{*}(\lambda)$ of $B_{\lambda, l}((-1 / 2 k, 0,0,0,0))$ for $\lambda>0$ such that

$$
\operatorname{Im} \rho_{l}^{*}\left(\lambda_{l}^{*}\right) \neq 0, \quad \operatorname{Re} \rho_{l}^{*}\left(\lambda_{l}^{*}\right)=0 \text { and } \frac{\partial}{\partial \lambda} \operatorname{Re} \rho_{l}^{*}\left(\lambda_{l}^{*}\right)>0
$$

This discussion is further enhanced for it can be shown that $\lambda_{l}^{*}$ is in fact a Hopf bifurcation value and there exists a limit cycle solution bifurcated from the equilibrium solution $(-1 / 2 k, 0,0,0,0)$. This is achieved by multiplying Eqs. (21) and (22) by $X_{3}$ and $X_{4}$ respectivly, to give

$$
\begin{aligned}
\frac{\mathrm{d} X_{3}^{2}}{\mathrm{~d} t} & =-2 a X_{3}^{2}-\frac{2 \lambda l k c}{a} X_{1} X_{4} X_{3}-\frac{2 \lambda l k d}{a} X_{1} X_{2} X_{3}, \\
\frac{\mathrm{d} X_{4}^{2}}{\mathrm{~d} t} & =-2 a X_{4}^{2}+\frac{2 \lambda l k c}{a} X_{1} X_{4} X_{3}+\frac{2 \lambda l k d}{a} X_{1} X_{5} X_{4} .
\end{aligned}
$$

The summation of these two equations implies

$$
\frac{\mathrm{d}\left(X_{3}^{2}+X_{4}^{2}\right)}{\mathrm{d} t}=-2 a\left(X_{3}^{2}+X_{4}^{2}\right)-\frac{2 \lambda l k d}{a} X_{1}\left(X_{2} X_{3}-X_{5} X_{4}\right)
$$

and by a similar process it follows from Eqs. (20-23) that 


$$
\begin{aligned}
\frac{\mathrm{d}\left(X_{2}^{2}+X_{5}^{2}\right)}{\mathrm{d} t} & =-2 b\left(X_{2}^{2}+X_{5}^{2}\right)-\frac{2 \lambda l k c}{b} X_{1}\left(X_{3} X_{2}-X_{4} X_{5}\right) \\
X_{4} \frac{\mathrm{d} X_{3}}{\mathrm{~d} t}-X_{3} \frac{\mathrm{d} X_{4}}{\mathrm{~d} t} & =-\frac{\lambda l k c}{a} X_{1}\left(X_{3}^{2}+X_{4}^{2}\right)-\frac{\lambda l k d}{a} X_{1}\left(X_{2} X_{4}+X_{3} X_{5}\right) \\
X_{5} \frac{\mathrm{d} X_{2}}{\mathrm{~d} t}-X_{2} \frac{\mathrm{d} X_{5}}{\mathrm{~d} t} & =-\frac{\lambda l k c}{b} X_{1}\left(X_{2} X_{4}+X_{3} X_{5}\right) \\
\frac{\mathrm{d}\left(X_{3} X_{5}+X_{4} X_{2}\right)}{\mathrm{d} t} & =\frac{\lambda l k c}{a} X_{1}\left(X_{2} X_{3}-X_{5} X_{4}\right)-2 d\left(X_{3} X_{5}+X_{4} X_{2}\right)
\end{aligned}
$$

Let us initially assume that $X_{1}(t)$ is convergent as $t$ tends to infinity. That is,

$$
\lim _{t \rightarrow \infty} X_{1}(t) \rightarrow X_{1, \lambda}
$$

for some constant $X_{1, \lambda}$. (This can always be validated by numerical experiments, see for example Fig. 1(b).) We thus have, by Eq. (19),

$$
\lambda l\left(X_{2}(t) X_{3}(t)-X_{4}(t) X_{5}(t)\right) \rightarrow 2 k X_{1, \lambda}+1 \text { as } t \rightarrow \infty,
$$

and by Eq. (30)

$$
\begin{aligned}
X_{2}(t) X_{4}(t)+X_{3}(t) X_{5}(t) & =\frac{\lambda l k c}{2 a d} X_{1}(t)\left(X_{2}(t) X_{3}(t)-X_{5}(t) X_{4}(t)\right) \\
& =\frac{k c}{2 a d} X_{1, \lambda}\left(2 k X_{1, \lambda}+1\right)
\end{aligned}
$$

when $t$ is sufficiently large. Under this latter condition, from Eqs. (19, 26-33) it follows that

$$
\begin{aligned}
X_{2} X_{3}-X_{4} X_{5} & =\frac{2 k X_{1, \lambda}+1}{\lambda l} \\
\frac{\mathrm{d}\left(X_{3}^{2}+X_{4}^{2}\right)}{\mathrm{d} t} & =-2 a\left(X_{3}^{2}+X_{4}^{2}\right)-\frac{2 k d}{a} X_{1, \lambda}\left(2 k X_{1, \lambda}+1\right), \\
X_{4} \frac{\mathrm{d} X_{3}}{\mathrm{~d} t}-X_{3} \frac{\mathrm{d} X_{4}}{\mathrm{~d} t} & =-\frac{\lambda l k c}{a} X_{1, \lambda}\left(X_{3}^{2}+X_{4}^{2}\right)-\frac{\lambda l k^{2} c}{2 a^{2}} X_{1, \lambda}^{2}\left(2 k X_{1, \lambda}+1\right), \\
\frac{\mathrm{d}\left(X_{2}^{2}+X_{5}^{2}\right)}{\mathrm{d} t} & =-2 b\left(X_{2}^{2}+X_{5}^{2}\right)-\frac{2 k c}{b} X_{1, \lambda}\left(2 k X_{1, \lambda}+1\right), \\
X_{5} \frac{\mathrm{d} X_{2}}{\mathrm{~d} t}-X_{2} \frac{\mathrm{d} X_{5}}{\mathrm{~d} t} & =-\frac{\lambda l k^{2} c^{2}}{2 a b d} X_{1, \lambda}^{2}\left(2 k X_{1, \lambda}+1\right) .
\end{aligned}
$$

With polar coordinate substitution

$$
X_{3}=r \cos \theta, X_{4}=r \sin \theta, X_{2}=s \cos \vartheta, X_{5}=s \sin \vartheta,
$$

this set of equations becomes

$$
\begin{aligned}
r s \cos (\theta+\vartheta) & =\frac{2 k X_{1, \lambda}+1}{\lambda l} \\
\frac{\mathrm{d} r^{2}}{\mathrm{~d} t} & =-2 a r^{2}-\frac{2 k d}{a} X_{1, \lambda}\left(2 k X_{1, \lambda}+1\right) \\
r^{2} \frac{\mathrm{d} \theta}{\mathrm{d} t} & =-\frac{\lambda l k c}{a} X_{1, \lambda} r^{2}-\frac{\lambda l k^{2} c}{2 a^{2}} X_{1, \lambda}^{2}\left(2 k X_{1, \lambda}+1\right),
\end{aligned}
$$




$$
\begin{aligned}
\frac{\mathrm{d} s^{2}}{\mathrm{~d} t} & =-2 b s^{2}-\frac{2 k c}{b} X_{1, \lambda}\left(2 k X_{1, \lambda}+1\right), \\
s^{2} \frac{\mathrm{d} \vartheta}{\mathrm{d} t} & =-\frac{\lambda l k^{2} c^{2}}{2 a b d} X_{1, \lambda}^{2}\left(2 k X_{1, \lambda}+1\right),
\end{aligned}
$$

and admits a stable periodic solution expressible as

or

$$
\begin{aligned}
r s \cos (\theta+\vartheta) & =\frac{2 k X_{1, \lambda}+1}{\lambda l} \\
r^{2} & =-\frac{k d}{a^{2}} X_{1, \lambda}\left(2 k X_{1, \lambda}+1\right) \\
r^{2} \frac{\mathrm{d} \theta}{\mathrm{d} t} & =-\frac{\lambda l k c}{a} X_{1, \lambda} r^{2}-\frac{\lambda l k^{2} c}{2 a^{2}} X_{1, \lambda}^{2}\left(2 k X_{1, \lambda}+1\right)
\end{aligned}
$$

or

$$
\begin{aligned}
\frac{\mathrm{d} \theta}{\mathrm{d} t} & =\frac{\lambda l k c(a-2 d)}{2 a d} X_{1, \lambda}, \\
s^{2} & =-\frac{k c}{b^{2}} X_{1, \lambda}\left(2 k X_{1, \lambda}+1\right), \\
s^{2} \frac{\mathrm{d} \vartheta}{\mathrm{d} t} & =-\frac{\lambda l k^{2} c^{2}}{2 a d b} X_{1, \lambda}^{2}\left(2 k X_{1, \lambda}+1\right)
\end{aligned}
$$

$$
\frac{\mathrm{d} \vartheta}{\mathrm{d} t}=\frac{\lambda l k c b}{2 a d} X_{1, \lambda}
$$

Finally, by Eq. (18), the limit cycle solution for $\lambda>\lambda_{l}^{*}$ with $1 \leq l<\sqrt{3} k$ can be expressed as follows:

$$
\begin{aligned}
r s \cos (\theta+\vartheta) & =\frac{2 k X_{1, \lambda}+1}{\lambda l} \\
r^{2} & =-\frac{k\left(l^{2}+5 k^{2}\right)}{\left(l^{2}+k^{2}\right)^{2}} X_{1, \lambda}\left(2 k X_{1, \lambda}+1\right), \\
\frac{\mathrm{d} \theta}{\mathrm{d} t} & =-\frac{\lambda l k\left(3 k^{2}-l^{2}\right)\left(l^{2}+9 k^{2}\right)}{2\left(l^{2}+k^{2}\right)\left(l^{2}+5 k^{2}\right)} X_{1, \lambda}, \\
s^{2} & =-\frac{k\left(3 k^{2}-l^{2}\right)}{\left(l^{2}+9 k^{2}\right)^{2}} X_{1, \lambda}\left(2 k X_{1, \lambda}+1\right), \\
\frac{\mathrm{d} \vartheta}{\mathrm{d} t} & =\frac{\lambda l k\left(3 k^{2}-l^{2}\right)\left(l^{2}+9 k^{2}\right)}{2\left(l^{2}+k^{2}\right)\left(l^{2}+5 k^{2}\right)} X_{1, \lambda} .
\end{aligned}
$$

This gives the supcritical Hopf bifurcation for Eq. (17) because this limit cycle is obviously the equilibrium solution $(-/ 2 k, 0,0,0,0)$ whenever $\lambda=\lambda_{l}^{*}$.

\section{Computational Results}

In this section, we provide computational results to strengthen the theoretical findings presented in Sects. 3-4. From the investigation of Sect. 4, we see that the projections of the limit cycle solutions of Eq. (17) onto the planes $\left(X_{3}, X_{4}\right)$ and $\left(X_{2}, X_{5}\right)$ are circles irrespective of the magnitude of $\lambda$. This phenomenon is valid for $\lambda \approx \lambda_{l}$ but 
when $\lambda$ diverges from $\lambda_{l}$, the truncation of the Navier-Stokes equations in the manner proposed becomes less reliable. This is because the infinite-dimensional dynamical system described by Eq. (6) was truncated to a five-dimensional dynamical system only represented by Eq. (17), and the truncation error is no longer small as $\lambda$ increases from $\lambda_{l}$. Therefore to assess the effect of the truncation, a further truncation model was adopted in which Eq. (6) with $k=l=1$ is reduced to a coupled set of thirteen ordinary differential equations. This of course provides an improved representation of the ideal situation (i.e. an infinite number of degrees of freedom) and it allows a relaxation of the condition $\lambda=\lambda_{l}$, such that $\lambda$ can now be chosen further from $\lambda_{l}$ before the truncation error dominates the numerical scheme. Furthermore, through this process we find that the error between the periodic Navier-Stokes flow described by Eq. (6) with $k=l=1$ and the limit cycle solution represented by the coupled set of thirteen ordinary differential equations is very much reduced. It should be noted that in this numerical example no second step bifurcation was found as $\lambda$ increases.

From the analysis described in Sects. 2-4, we note that there is little significant difference amongst the topological structure of the periodic flows defined by Eq. (6) for the case $1 \leq l<\sqrt{3} k$ with $k \geq 1$. By way of an example, therefore, we only consider the case $k=l=1$.

Let us begin with the computation of Eq. (17) with $k=l=1$ associated with the five-dimensional dynamical system. That is, the coupled set of equations

$$
\begin{aligned}
\frac{\mathrm{d} X_{1}}{\mathrm{~d} t} & =-4 X_{1}+2 \lambda X_{3} X_{2}-2 \lambda X_{4} X_{5}-2, \\
\frac{\mathrm{d} X_{2}}{\mathrm{~d} t} & =-10 X_{2}-\frac{1}{5} \lambda X_{1} X_{3}, \\
\frac{\mathrm{d} X_{3}}{\mathrm{~d} t} & =-2 X_{3}-\lambda X_{1} X_{4}-3 \lambda X_{1} X_{2}, \\
\frac{\mathrm{d} X_{4}}{\mathrm{~d} t} & =-2 X_{4}+\lambda X_{1} X_{3}+3 \lambda X_{1} X_{5}, \\
\frac{\mathrm{d} X_{5}}{\mathrm{~d} t} & =-10 X_{5}+\frac{1}{5} \lambda X_{1} X_{4},
\end{aligned}
$$

associated with the Hopf bifurcation value

$$
\lambda_{1}^{*}=120 / \sqrt{83} \approx 13.3,
$$

which is derived from Eq. (25).

Let us denote $\mathbf{X}=\left(X_{1}, X_{2}, X_{3}, X_{4}, X_{5}\right)$ and the right-hand side of Eq. (35) by $f_{1, \lambda}(\mathbf{X})$. The resultant equation can be discretised by the three-step Adams-Bashforth method (see Lambert (1991)) to give

$$
\mathbf{X}_{n+1}=\mathbf{X}_{n}+\frac{h}{12}\left(23 f_{1, \lambda}\left(\mathbf{X}_{n}\right)-16 f_{1, \lambda}\left(\mathbf{X}_{n-1}\right)+5 f_{1, \lambda}\left(\mathbf{X}_{n-2}\right)\right)
$$

where the step length $h=0.0002$, and for demonstration purposes we arbitrarily choose a Reynolds number $\lambda=15$ (i.e. close to the Hopf bifurcation value $\lambda_{1}^{*}$ ) or $\lambda=$ 210. Since the periodic solution is stable, any initial value outside the stable manifold of the equilibrium solution $(-1 / 2,0,0,0,0)$ generates a trajectory approaching the limit cycle solution. We thus need only choose the initial value

$$
\mathbf{X}_{0}=(-1 / 2,0,1,0,0)
$$



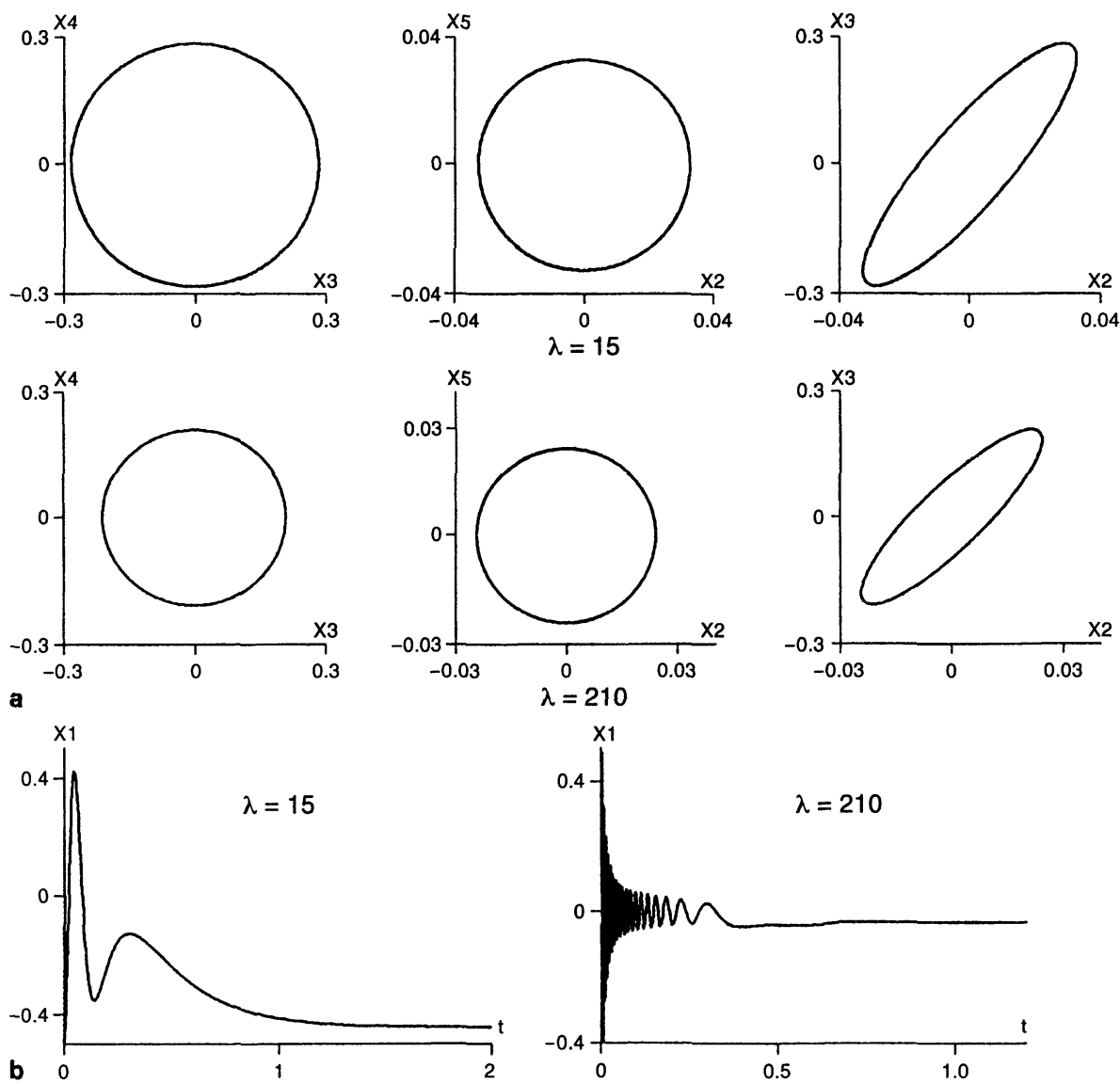

Fig. 1. (a) Phase portraits of the limit cycle solutions derived from the five-dimensional truncation model $(k=l=1)$ for Reynolds number $\lambda=15$ and $\lambda=210$. The Hopf bifurcation occurs at $\lambda=\lambda_{1}^{*} \approx 13.3$. (b) Demonstration of the convergence coordinate $X_{1}(t)$ as $t \rightarrow \infty$ (see Eq. (31)).

The computational results for this example are displayed in Fig. 1, which shows that the assumption of Eq. (31) is satisfied, and

$$
X_{1, \lambda}=-0.439057 \text { for } \lambda=15 \text { and } X_{1, \lambda}=-0.031361 \text { for } \lambda=210 .
$$

The limit cycle solutions illustrated in Fig. 1 coincide with those presented by Eq. (34) and the corresponding phase portraits on the $\left(X_{3}, X_{4}\right)$ and $\left(X_{2}, X_{5}\right)$ planes are

$$
\begin{aligned}
& X_{3}^{2}+X_{4}^{2}=r^{2}=0.283324^{2} \text { and } X_{2}^{2}+X_{5}^{2}=s^{2}=0.032715^{2} \text { for } \lambda=15, \\
& X_{3}^{2}+X_{4}^{2}=r^{2}=0.209978^{2} \text { and } X_{2}^{2}+X_{5}^{2}=s^{2}=0.024246^{2} \text { for } \lambda=210 .
\end{aligned}
$$

To proceed to a more detailed representation of the dynamical system defined in Eq. (6) with $k=l=1$ by a coupled set of equations greater in number than 5 , it is beneficial to note that every solution of Eq. (6) with $k=l=1$ is of the following form: 


$$
\begin{aligned}
\psi=\sum_{n=1}^{\infty} \xi_{n}(t) & \cos 2 n y+\sum_{m=1}^{\infty} \sum_{n=-\infty}^{\infty} \eta_{m, n}(t) \cos (2 m x-x+2 n y+y) \\
& +\sum_{m=1}^{\infty} \sum_{n=-\infty}^{\infty} \zeta_{m, n}(t) \cos (2 m x+2 n y) .
\end{aligned}
$$

This Navier-Stokes flow is excited by the spatial force $2 \cos 2 y$, and the Hopf bifurcation stems from the term $\sum_{n=-\infty}^{\infty} \eta_{1, n} \cos (x+2 n y+y)$, which then influences the terms $\sum_{n=-\infty}^{\infty} \zeta_{1, n} \cos (2 x+2 n y)$ and $\sum_{n=1}^{\infty} \xi_{n} \cos 2 n y$. Furthermore, the modes $\cos (x+2 n y+y)$ and $\cos (2 x+2 n y)$ have limited symmetry with the modes $\cos (x-2 n y-y)$ and $\cos (2 x-2 n y)$ respectively, and by numerical experiments $\xi_{n}$, $\eta_{1, n}, \eta_{1,-n-1}, \zeta_{1, n}$ and $\zeta_{1,-n}$ can be shown to be of order $10^{-n-1}$ for $n \geq 0$. For these reasons, this Navier-Stokes flow can be adequately approximated by a function in the following form:

$$
\sum_{n=1}^{N} \xi_{n}(t) \cos 2 n y+\sum_{n=-N-1}^{N} \zeta_{1, n}(t) \cos (x+2 n y+y)+\sum_{n=-N}^{N} \eta_{1, n}(t) \cos (2 x+2 n y)
$$

for $N>1$. Here, for simplification, we take $N=2$. Therefore, by the previous reasoning, Eq. (6) with $k=l=1$ is projected onto the thirteen-dimensional space

$$
\begin{gathered}
\operatorname{span}\{\cos 2 y, \cos 4 y, \cos (x-5 y), \cos (x-3 y), \cos (x-y), \cos (x+y), \cos (x+3 y), \\
\cos (x+5 y), \cos (2 x-4 y), \cos (2 x-2 y), \cos 2 x, \cos (2 x+2 y), \cos (2 x+4 y)\}
\end{gathered}
$$

and, after rearrangement, the Navier-Stokes flow is truncated to the following form:

$$
\begin{aligned}
\psi(t)= & \sum_{j=1}^{2} X_{j}(t) \cos 2 j y+\sum_{n=3}^{8} X_{n}(t) \cos (x+2 n y-11 y) \\
& +\sum_{m=9}^{13} X_{m}(t) \cos (2 x+2 m y-22 y)
\end{aligned}
$$

Letting

$$
\begin{aligned}
& \phi_{j}=\sin 2 j y, \phi_{n}=\sin (x+2 n y-11 y), \phi_{m}=\sin (2 x+2 m y-22 y), \\
& \psi_{\jmath}=\cos 2 j y, \psi_{n}=\cos (x+2 n y-11 y), \psi_{m}=\cos (2 x+2 m y-22 y)
\end{aligned}
$$

for $j=1,2, n=3, \ldots, 8$ and $m=9, \ldots, 13$, we have

$$
\begin{aligned}
-\partial_{y} \psi & =\sum_{j=1}^{2} 2 j X_{\jmath}(t) \phi_{\jmath}+\sum_{n=3}^{8}(2 n-11) X_{n}(t) \phi_{n}+\sum_{m=9}^{13}(2 m-22) X_{m}(t) \phi_{m}, \\
\partial_{x} \Delta \psi & =\sum_{n=3}^{8}\left(1+(2 n-11)^{2}\right) X_{n}(t) \phi_{n}+\sum_{m=9}^{13} 2\left(4+(2 m-22)^{2}\right) X_{n}(t) \phi_{m}, \\
-\partial_{x} \psi & =\sum_{n=3}^{8} X_{n}(t) \phi_{n}+\sum_{m=9}^{13} 2 X_{m}(t) \phi_{m}
\end{aligned}
$$




$$
\begin{aligned}
\partial_{y} \Delta \psi= & \sum_{i=1}^{2} 8 i^{3} X_{i}(t) \phi_{i}+\sum_{n=3}^{8}(2 n-11)\left(1+(2 n-11)^{2}\right) X_{n}(t) \phi_{n} \\
& +\sum_{m=9}^{13}(2 m-22)\left(4+(2 m-22)^{2}\right) X_{m}(t) \phi_{m}
\end{aligned}
$$

and so, after the necessary manipulations, the non-linear contribution is given by

$$
\begin{aligned}
\partial_{y} \psi & \partial_{x} \Delta \psi-\partial_{x} \psi \partial_{y} \Delta \psi \\
= & \sum_{j=1}^{2}\left(\sum_{n=3+j}^{8} 4 j^{2}(2 n-j-11) X_{n} X_{n-j}+\sum_{m=9+j}^{13} 8 j^{2}(2 m-j-22) X_{m} X_{m-j}\right) \psi_{j} \\
& +\sum_{j=1}^{2} \sum_{n=3}^{8-j} j\left(4 j^{2}-1-(2 n+2 j-11)^{2}\right) X_{j} X_{n+j} \psi_{n} \\
& -\sum_{j=1}^{2} \sum_{n=3+j}^{8} j\left(4 j^{2}-1-(2 n-2 j-11)^{2}\right) X_{j} X_{n-j} \psi_{n} \\
& +\sum_{m=9}^{13} \sum_{3 \leq m-n, n \leq 8}(m-2 n)((11-2 n)(4 m-2 n-33)-3) X_{m} X_{m-n} \psi_{n} \\
& +\sum_{j=1}^{2} \sum_{m=9}^{13-j} 8 j\left(j^{2}-1-(m+j-11)^{2}\right) X_{j} X_{m+j} \psi_{m} \\
& -\sum_{j=1}^{2} \sum_{m=9+j}^{13} 8 j\left(j^{2}-1-(m-j-11)^{2}\right) X_{j} X_{m-j} \psi_{m} \\
& +\sum_{m=9}^{13} \sum_{3 \leq m-n, n \leq 8}(m-2 n)\left(1+(2 n-11)^{2}\right) X_{n} X_{m-n} \psi_{m}+E
\end{aligned}
$$

where $E$ is a term orthogonal to the thirteen-dimensional space. Eq. (6) is thus truncated to the following coupled set of thirteen ordinary differential equations:

$$
0=\sum_{j=1}^{13} \int_{\mathscr{T}^{2}}\left(\frac{\mathrm{d} \psi}{\mathrm{d} t}-\Delta \psi-f_{\lambda}(\psi)\right) \psi_{j} \mathrm{~d} x \mathrm{~d} y \psi_{j}
$$

which on expansion gives

$$
\begin{aligned}
0= & \sum_{j=1}^{13} \frac{\mathrm{d} X_{j}}{\mathrm{~d} t} \psi_{j}+\sum_{j=1}^{2} 4 j^{2} X_{j} \psi_{j}+\sum_{n=3}^{8}\left(1+(2 n-11)^{2}\right) X_{n} \psi_{n} \\
& +\sum_{m=9}^{13}\left(4+(2 m-22)^{2}\right) X_{m} \psi_{m}+2 \psi_{1}
\end{aligned}
$$



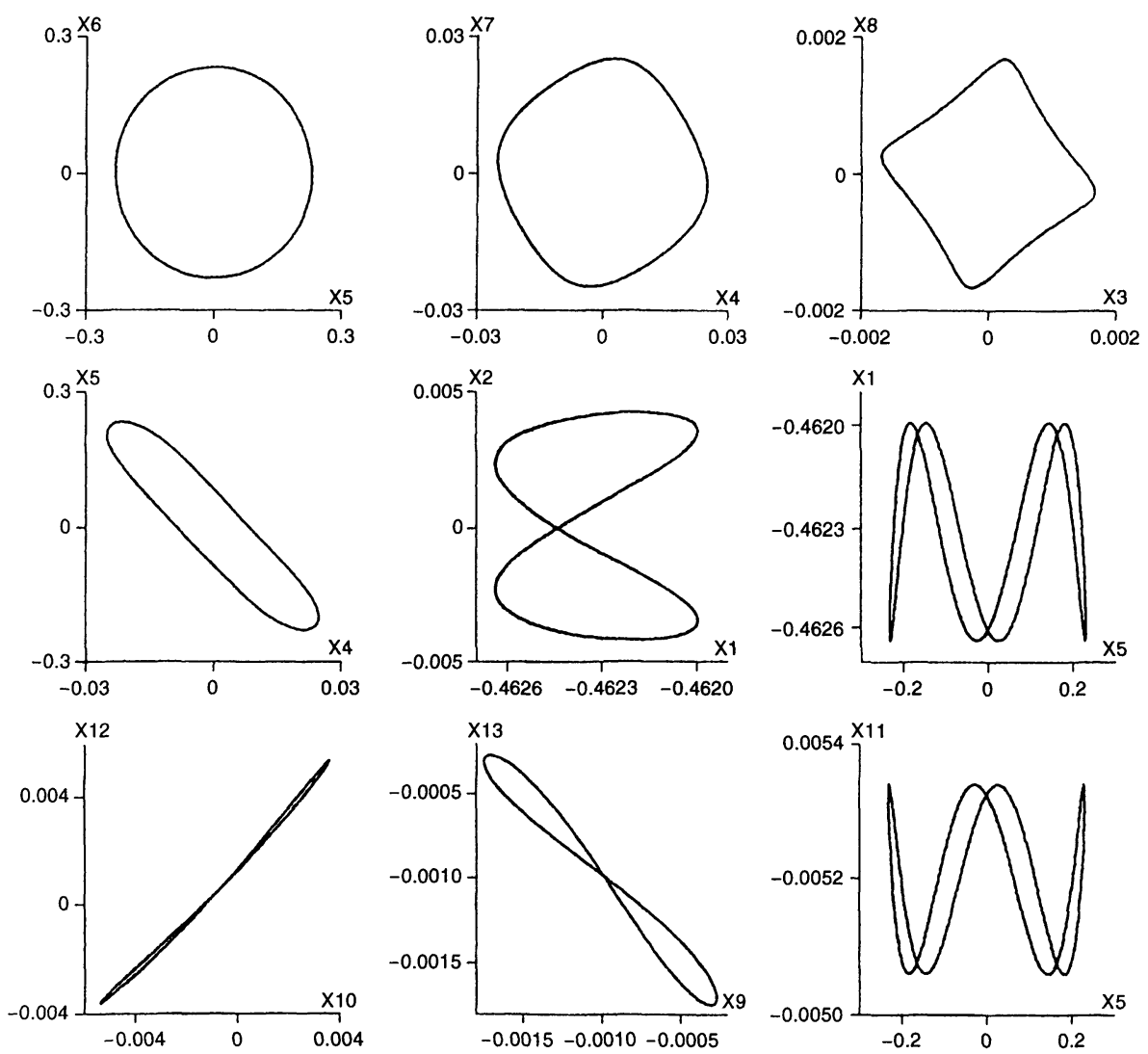

Fig. 2. Phase portraits of the limit cycle solution derived from the thirteen-dimensional truncation model $(k=l=1)$ for Reynolds number $\lambda=15$ (Hopf bifurcation value $\lambda_{1}^{* *} \approx 14$ ).

$$
\begin{aligned}
& +\lambda \sum_{j=1}^{2}\left(\sum_{n=3+j}^{8}(2 n-j-11) X_{n} X_{n-j}+\lambda \sum_{m=9+j}^{13} 2(2 m-j-22) X_{m} X_{m-j}\right) \psi_{j} \\
& +\lambda \sum_{j=1}^{2} \sum_{n=3}^{8-j} \frac{j\left(4 j^{2}-1-(2 n+2 j-11)^{2}\right)}{1+(2 n-11)^{2}} X_{j} X_{n+j} \psi_{n} \\
& -\lambda \sum_{j=1}^{2} \sum_{n=3+\jmath}^{8} \frac{j\left(4 j^{2}-1-(2 n-2 j-11)^{2}\right)}{1+(2 n-11)^{2}} X_{j} X_{n-j} \psi_{n} \\
& +\lambda \sum_{m=9}^{13} \sum_{3 \leq m-n, n \leq 8} \frac{(m-2 n)((11-2 n)(4 m-2 n-33)-3)}{1+(2 n-11)^{2}} X_{m} X_{m-n} \psi_{n} \\
& +\lambda \sum_{j=1}^{2} \sum_{m=9}^{13-\jmath} \frac{2 j\left(j^{2}-1-(m+j-11)^{2}\right)}{1+(m-11)^{2}} X_{\jmath} X_{m+j} \psi_{m}
\end{aligned}
$$




$$
\begin{aligned}
& -\lambda \sum_{j=1}^{2} \sum_{m=9+j}^{13} \frac{2 j\left(j^{2}-1-(m-j-11)^{2}\right)}{1+(m-11)^{2}} X_{j} X_{m-j} \psi_{m} \\
& +\lambda \sum_{m=9}^{13} \sum_{3 \leq m-n, n \leq 8} \frac{(m-2 n)\left(1+(2 n-11)^{2}\right)}{4+4(m-11)^{2}} X_{n} X_{m-n} \psi_{m}
\end{aligned}
$$

The rewriting of this coupled set of equations in the following form:

$$
\frac{\mathrm{d} \mathbf{X}}{\mathrm{d} t}=f_{2, \lambda}(\mathbf{X}), \mathbf{X}=\left(X_{1}, \ldots, X_{13}\right)
$$

leads itself to a discretisation by the 4-step Adams-Bashforth method to give

$$
\mathbf{X}_{n+1}=\mathbf{X}_{n}+\frac{h}{24}\left(55 f_{2, \lambda}\left(\mathbf{X}_{n}\right)-59 f_{2, \lambda}\left(\mathbf{X}_{n-1}\right)+37 f_{2, \lambda}\left(\mathbf{X}_{n-2}\right)-9 f_{2, \lambda}\left(\mathbf{X}_{n-3}\right)\right),
$$

where a step length $h=0.0002$ is again chosen. To illustrate the variation of the limit cycle solution as the Reynolds number $\lambda$ increases, Eq. (36) at Reynolds numbers
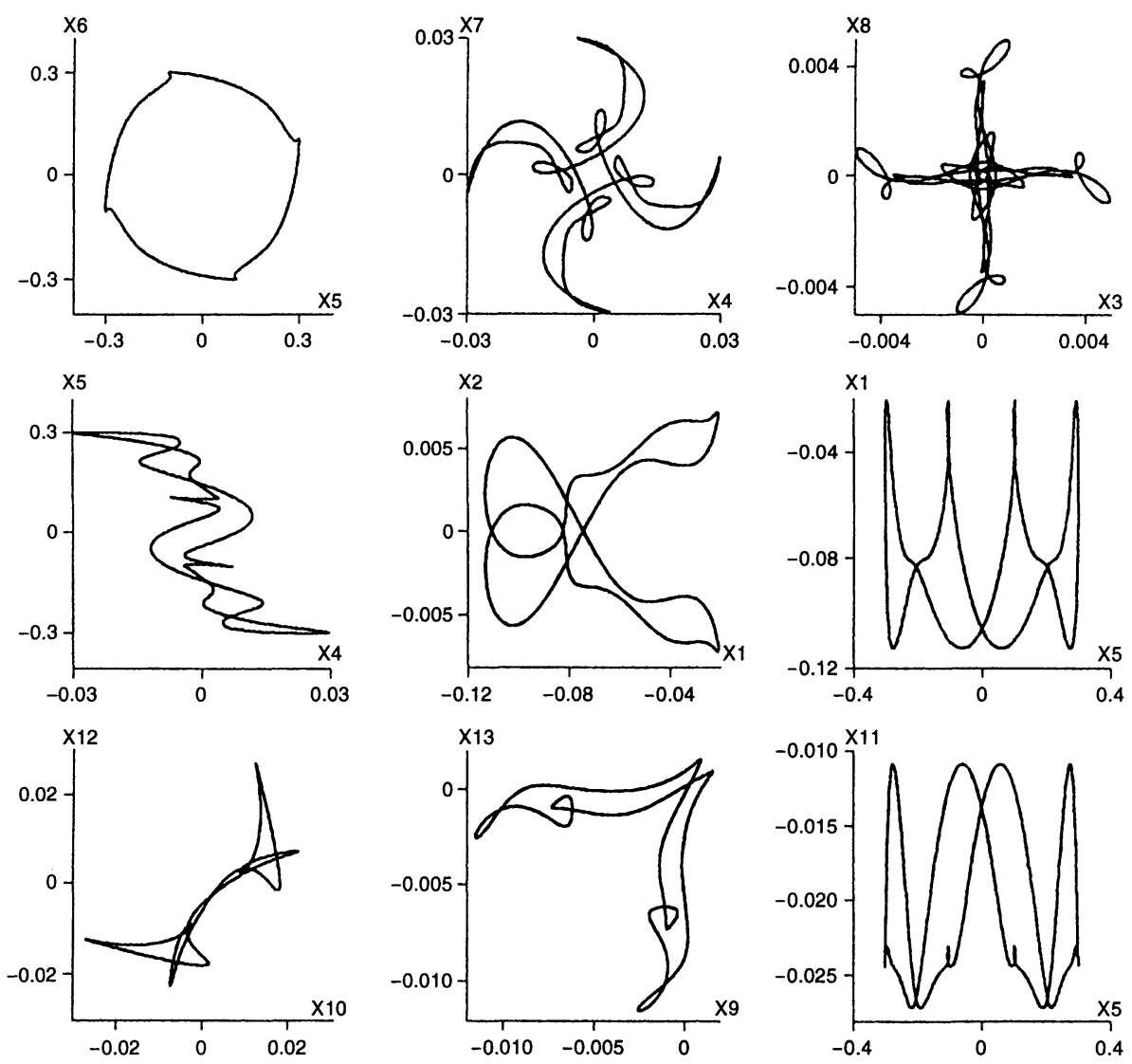

Fig. 3. Phase portraits of the limit cycle solution derived from the thirteen-dimensional truncation model $\left(k=l=1\right.$ ) for Reynolds number $\lambda=210$ (Hopf bifurcation value $\lambda_{1}^{* *} \approx 14$ ). 
$\lambda=15,21,108,140,180$ and 210 were examined. Similar to the five-dimensional example, we need only choose the initial value $\mathbf{X}$ with $X_{1}=-1 / 2, X_{5}=1$ and $X_{n}=0$ for $n \neq 1,5$. From numerical experiments we find that the Hopf bifurcation value $\lambda_{1}^{* *}$ for this set of equations is near 14 as compared to $\lambda_{1}^{*}(\approx 13.3)$ for the five-dimensional case. The computational results for a selection of symmetrical phase portraits of the limit cycle solutions with $\lambda=15$ and 210 are displayed in Figs. 2-3.

These figures together with the intermediate calculations at $\lambda=21,108,140,180$ illustrate that Eq. (36) provides a satisfactory approximation of Eq. (6), and the limit cycle solutions of Eq. (36) become more and more complicated in form as $\lambda$ increases. Fig. 2 illustrates the case of $\lambda$ close to $\lambda_{1}^{* *}$ (i.e. $\lambda=15, \lambda_{1}^{* *} \approx 14$ ), whereas Fig. 3 shows the effect of $\lambda$ substantially removed from $\lambda_{1}^{* *}$ (i.e. $\lambda=210, \lambda_{1}^{* *} \approx 14$ ). Futhermore the evidence presented in Figs. 2-3 also suggests that the periodic NavierStokes flows of Eq. (6) are stable irrespective of the magnitude of the Reynolds number $\lambda$.

\section{References}

[AM] Arnold, V. I., Meshalkin, L. D.: A. N. Kolmogorov's seminar on selected problems of analysis (1958-1959). Russ. Math. Surv. 15 (1), 247-250 (1960)

[FGN] Franceschini, V., Giberti, C., Nicolini, M.: Common periodic behaviour in larger truncations of the Navier-Stokes equations. J. Stat. Phys. 50, 879-896 (1988)

[GH] Guckenheimer, J., Holmes, P.: Nonlinear oscillations, dynamical systems, and bifurcations of vector fields. Berlin: Springer, 1983

[Iud] Iudovich, V. I.: Example of the generation of a secondary stationary or periodic flow when there is loss of stability of the laminar flow of a viscous incompressible fluid. J. Math. Mech. 29(4), 587-603 (1965)

[Iud] Iudovich, V. I.: The onset to auto-oscillations in a fluid. J. Math. Mech. 35(3), 453-467 (1971)

[Lam] Lambert, J. D.: Numerical methods for ordinary differential systems. Chichester: Wiley, 1991

[Lan] Landau, L.: On the problem of turbulence. Comptes Rend. Acad. Sci. USSR 44, 311-316 (1944)

[Liu] Liu, V. X.: An example of instability for the Navier-Stokes equations on the 2-dimensional torus. Commun. Partial Differential Eqs. 17, 1995-2012 (1992a)

[Liu] Liu, V. X.: Instability for the Navier-Stokes equations on the 2-dimensional torus and a lower bound for the Hausdorff dimension of their global attractors. Commun. Math. Phys. 147, 217-230 (1992b)

[Mar] Marchioro, C.: An example of absence of turbulence for any Reynolds number. Commun. Math. Phys. 105, 99-106 (1986)

[MM] Marsden, J. E., McCracken, M.: The Hopf bifurcation and its applications. Berlin: Springer, 1976

[Mas] Massey, M. S.: A basic course in algebraic topology. Berlin: Springer, 1991

[MS] Meshalkin, L. D., Sinai, Ya. G.: Investigation of the stability of a stationary solution of a system of equations for the plane movement of an incompressible viscous fluid. J. Math. Mech. 19(9), 1700-1705 (1961)

[NRT] Newhouse, S. E., Ruelle, D., Takens, F.: Occurrence of strange axiom-A attractors near quasiperiodic flows on $T^{n}, n \geq 3$. Commun. Math. Phys. 64, 35-40 (1978)

[OS] Okamoto, H., Shoji, M.: Bifurcation diagrams in Kolmogorov's problem of viscous incompressible fluid on 2-D flat tori. Kyoto Univ. Res. Inst. Math. Sci. 767, 29-51 (1991)

[RT] Ruelle, D., Takens, F.: On the nature of turbulence. Commun. Math. Phys. 20, 167-192 (1971)

[TS] Thompson, J. M. T., Stewart H. B.: Nonlinear dynamics and chaos. Chichester: Wiley, 1986

[Tri] Triebel, H.: Interpolation theory, function spaces, differential operators. Amsterdam: North-Holland, 1978

Communicated by Ya. G. Sinai

This article was processed by the author using the LTT $_{\mathrm{E}} \mathrm{X}$ style file pljourl from Springer-Verlag. 
